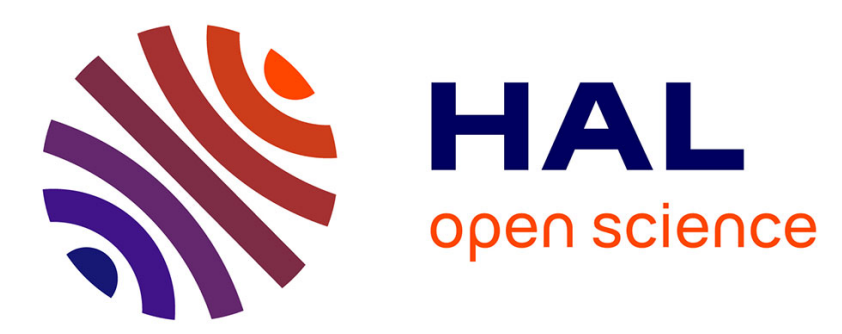

\title{
Morphogenesis of surfaces with planar lines of curvature and application to architectural design
} Romain Mesnil, Cyril Douthe, Olivier Baverel, Bruno Léger

\section{To cite this version:}

Romain Mesnil, Cyril Douthe, Olivier Baverel, Bruno Léger. Morphogenesis of surfaces with planar lines of curvature and application to architectural design. Automation in Construction, 2018, 95, pp.129 - 141. 10.1016/j.autcon.2018.08.007 . hal-01899094

\section{HAL Id: hal-01899094 https://hal.science/hal-01899094}

Submitted on 19 Oct 2018

HAL is a multi-disciplinary open access archive for the deposit and dissemination of scientific research documents, whether they are published or not. The documents may come from teaching and research institutions in France or abroad, or from public or private research centers.
L'archive ouverte pluridisciplinaire HAL, est destinée au dépôt et à la diffusion de documents scientifiques de niveau recherche, publiés ou non, émanant des établissements d'enseignement et de recherche français ou étrangers, des laboratoires publics ou privés. 


\title{
Morphogenesis of surfaces with planar lines of curvature and application to architectural design
}

\author{
Romain Mesnil ${ }^{\mathrm{a}, \mathrm{b}, *}$, Cyril Douthe ${ }^{\mathrm{a}}$, Olivier Baverel ${ }^{\mathrm{a}}$, Bruno Léger $^{\mathrm{b}}$ \\ ${ }^{a}$ Laboratoire Navier, UMR 8205, École des Ponts, IFSTTAR, CNRS, UPE, Marne-La-Vallée, France \\ ${ }^{b}$ Bouygues Construction SA, Guyancourt, France
}

\begin{abstract}
This article presents a methodology to generate surfaces with planar lines of curvature from two or three curves and tailored for architectural design. Meshing with planar quadrilateral facets and optimal offset properties for the structural layout are guaranteed. The methodology relies on the invariance of circular meshes by spherical inversion and discrete Combescure transformations, and uses parametrisation of surfaces with cyclidic patches. The shapes resulting from our methodology are called super-canal surfaces by the authors, as they are an extension of canal surfaces. An interesting connection to shell theory is recalled, as the shapes proposed in this paper are at equilibrium under uniform normal loading. Some applications of these shapes to architecture are shown.
\end{abstract}

Keywords: super-canal surface, fabrication-aware design, cylidic net, architectural geometry, structural morphogenesis, façade

\section{1. Introduction}

\subsection{Constructive geometry in architecture}

The construction of architectural shapes is subject to technological constraints that highly impact the economy of the cladding and structure. The study that aims at expressing technological requirements as geometrical constraints is often referred to as fabrication-aware design in the computer science community, whereas architects or engineers speak of shape rationalization or constructive geometry. This topic, takes root in the eighteenth century and stereotomy, and the work of Gaspard Monge[1].

In glass or metal envelopes, the planarity of the panels is regarded as one of the most significant aspect in the design of technogically-feasible solutions, and motivated the creation of tailor-made morphogenesis strategies by engineering office Schlaich Bergermann und Partner [2] and

* Corresponding author

E-mail: romain.mesnil@enpc.fr later by Gehry Technologies [3]. Triangular meshes are always covered with planar facets, but their high node valence makes the fabrication of the structure complicated [4]. They are also considered less transparent than quadrilateral layouts [3]. Developable panels are also of interest because cold-bending technologies for glass can be used at a reasonable cost, as illustrated by some projects of engineering office RFR $[5,6]$.

The geometry of the supporting structure is another indicator of the complexity of fabrication in free-form architecture. The most economical solution is to build with planar beams that meet exactly along axes. This topic is well-known by gridshell builders [7] and is covered from a mathematical perspective in [8], with a tool called 'mesh parallelism'. Building a support structure with planar beams implies indeed the existence of a mesh which has all its edges parallel to the initial mesh.

These two construction constraints (planarity of panels 
and planarity of beams) can be integrated in the design 72 of free-form architecture, either in top-down $[4,6,9]$ or in bottom-up approaches [10, 11, 12, 13, 14]. The latter approaches generate design spaces where the fabrication requirements are fulfilled. They offer thus the possibility to integrate constraints of a different nature early in the design process, like structural behaviour or energy consumption. This is particularly important in the context of architectural design: fabrication is only one of the many criteria that should be rationalised or optimised in a building envelope.

\subsection{Geometrically-constrained shape generation}

A natural way to deal with construction constraints is ${ }^{73}$ to generate a design-space of shapes that satisfy the most ${ }^{74}$ critical fabrication-constraints. This approach, known as ${ }^{75}$ "geometrically-constrained design strategy" [15] has been ${ }^{76}$ used extensively in the history of architecture. Methods ${ }^{77}$ that guarantee planar quads include surfaces of revolution, ${ }^{78}$ surfaces of translation [2], scale-trans surfaces [3], mould- ${ }^{79}$ ing and Monge surfaces $[16,17,18]$. These surfaces can be generated using two curves and a rule of transformation, ${ }^{81}$ either translation or sweeping along Bishop's frame. The ${ }^{82}$ designer controls the overall shape and its discretisation ${ }^{83}$ simultaneously, which makes all these shapes easily un- ${ }^{84}$ derstandable and usable [19]. Accordingly, geometrically- ${ }^{85}$ constrained approches using two curves like surfaces of ${ }^{86}$ translation are very popular in the community of struc- ${ }^{87}$ tural engineers [20].

Table 1 shows the correspondance between shape gen- ${ }^{89}$ eration techniques using two or three curves and their ${ }^{90}$ fabrication-aware counterpart. For example extrusion ${ }^{91}$ along a curve that yields surfaces of translation and sur- ${ }^{92}$ faces of revolution are good examples of fabrication-aware ${ }^{93}$ shapes. Monge surfaces, that can be generated by sweeping a planar curve called generatrix along a rail curve, are also very interesting for architectural shape design. It can be noticed that the sweep 2 rails command has no fabrication-aware equivalent.

\begin{tabular}{|c|c|}
\hline CAD generation process & Fabrication-aware shape \\
\hline Extrusion along curve & Surface of translation \\
\hline Revolve & \begin{tabular}{c} 
Surface of revolution \\
\hline Rail Revolve
\end{tabular} \\
\hline Sweep 1 rail & $\begin{array}{c}\text { Monge surface* } \\
\text { Isoradial mesh }\end{array}$ \\
\hline Sweep 2 rails & - \\
\hline
\end{tabular}

Table 1: Kinematic method to generate free-form surfaces and their fabrication-aware equivalent, surfaces marked with asterisks are subject to additional constraints.

The objective of this work is thus to enrich the design space accessible with geometrically-constrained design strategies by proposing new shapes constructed from two and three curves. The shapes can be generated in realtime on standard computers, which eases the exploration of this design space.

The second section of this paper discusses thus the general methodology that generates super-canal surfaces, a new family of shapes for fabrication-aware design in architecture, as well as a new algorithm for the fast computation of parallel meshes. Applying the results of [21], we also show that super-canal surfaces are remarkable with respect to shell theory: their lines of curvatures are lines of principal stress under uniform normal loading. This work thus meets fabrication with equilibrium, two major aspects of architectural design. A new method for the generation of canal surfaces from two contour curves is presented in Section 4. The fifth Section introduces some inverse problems solved in with super-canal surface. A brief discussion and conclusion sum up the contributions of the present article. 


\subsection{Möbius geometry and cyclidic nets}

The present methodology for shape generation relies on a more general framework proposed recently for architectural design [22] in the following of previous work developped in $[23,24,25,26]$. The main concept is to link discrete objects, namely circular meshes, with a smooth underlying surface. All the shapes are thus described as coarse circular meshes, which support portions of Dupin's cyclides. Among remarkable features of cyclides, one may mention that their lines of curvature are circles and that a patch delimited by four lines of curvature on a Dupin cyclide has its four vertices inscribed within a circle. The formal potential of this framework is shown in [26], where various fitting problems on complex shapes are solved.

Cyclidic nets provide thus a natural way to cover complex shapes with circular quadrilateral meshes. Moreover, as transformations mapping circular quadrilaterals to circular quadrilaterals also preserve cyclidic nets and the underlying parametrisation, such transformations are of particular interest. Two of those will be studied in the following: Möbius transformations in Section 2.3 and Combescure transformations in Section 2.4. Starting from surfaces easily described with cyclidic nets, the application of these transformations creates new shapes for fabrication-aware design in architecture.

\subsection{Geometrical properties of canal surface}

Canal surfaces are a fundamental family of surfaces in the context of Möbius geometry, as this family is indeed invariant by Möbius transformations. Canal surfaces are ${ }^{145}$ defined as envelopes of spheres. They are commonly used ${ }^{146}$ as blending surfaces, either by using contour curves [27] or $^{147}$ by joining spheres that can be manipulated by the designer [28].

Alternatively, canal surfaces can be defined as surfaces such that lines of curvatures are circles. The invariance of canal surfaces under Möbius transformations is obvious, because inversions preserve lines of curvatures and circles. Another way to look at it is that Möbius transformations preserve both spheres and angles, therefore an envelope of spheres is preserved by inversions. Notice that the shapegeneration of canal surfaces with Dupin cyclides has been studied in [29]. We use an optimisation approach introduced in [26] to obtain shapes with smooth parametrisations.

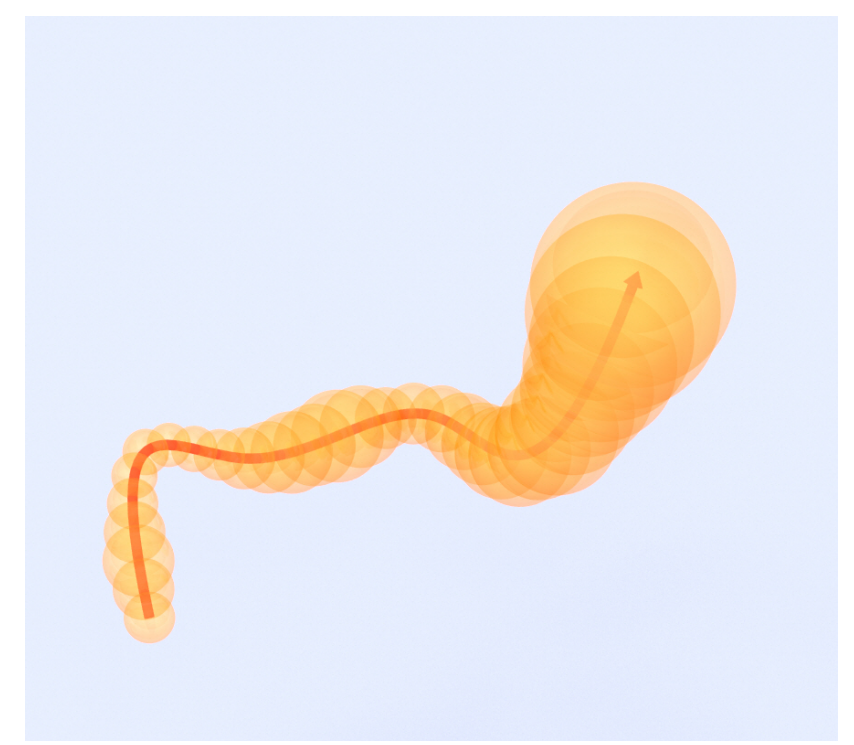

Figure 1: Canal surface as an envelope of spheres whose centers are on a curve.

These surfaces are very easily parameterised by cyclidic patches, as Dupin cyclides are particular cases of canal surfaces. The $C^{1}$ continuity and generalisation to $C^{0}$ continuity is insured by reflections according to the method described in [25].

\subsection{Möbius transformations}

The transformation at the core of the framework using cyclidic nets is the Möbius transformation or inversion, which is a very simple non-linear map. We recall here some of its elementary properties, and introduce the notations used in the following of this paper.

Möbius transformations preserve locally angles, and are thus conformal maps. They also preserves circles. Möbius transformations are compositions of translation, 
scaling and spherical inversions. The latter transforma-174 tion is defined by a center and a ratio. Consider a point ${ }_{175}$ $\mathbf{C}$, later called center of inversion, and a real number $k .176$ The inversion of center $\mathbf{C}$ and ratio $k$ applied to a point ${ }_{177}$ $\mathbf{M}$ is a point $\mathbf{M}^{\prime}$ defined by the well-known equation: ${ }_{178}$

$$
\mathrm{CM}^{\prime}=\frac{k}{\|\mathbf{C M}\|^{2}} \cdot \mathbf{C M}
$$

In the complex plane, the inversion of ratio $k$ with center

$\mathbf{C}$ (complex number $z_{C}$ ) reads as:

$$
f_{k, \mathbf{C}}(z)=z_{C}+\frac{k}{\bar{z}-\overline{z_{C}}}
$$

\section{1}

An elementary property of inversions is that they are involutions, which means that Möbius transformations are their own inverse transformations. This property is used in many applications shown in this paper (see Section 4). It can finally be noticed that the ratio $k$ is nothing more than a scaling factor. The position of the point $\mathbf{C}$ is the parameter that has a true impact on the shape deformation.

\subsection{Combescure transformations}

It has just been seen that Möbius transformations allow to modify the overall appearance of circular meshes by preserving the circumcircles of all quads. Another transformation that has the same property is the mesh parallelism transformation. Two meshes are said parallels if they have the same connectivity and if all their edges are parallel. The transformation mapping one mesh to the other is called a Combescure transformation [8]. By definition, Combescure transformations preserve discrete angles. Therefore they map circular meshes to circular meshes. Combined with Möbius transformations, they offer a range of possibilities to deform circular meshes.

Two meshes related by a Combescure transformation, with respective edges $\left(\mathbf{e}_{\mathbf{i}}\right)$ and $\left(\mathbf{e}_{\mathbf{i}}^{\prime}\right)$, have to satisfy a linear equation:

$$
\forall i, \mathbf{e}_{\mathbf{i}} \wedge \mathbf{e}_{\mathbf{i}}^{\prime}=\mathbf{0}
$$

Solutions for this equation are usually found using Singular Value Decomposition (SVD) [8]. We introduce here a different original approach, restricted to quadrilateral meshes, but that offers a better performance than SVD. This technique takes inspiration from the one employed in [14], which is applied to the form-finding of planarquadrilaterals meshes.

\subsection{Efficient computation of Combescure transformations}

Let us consider two parallel quadrilaterals, like the ones shown in Figure 2. Up to a translation, prescribing the lengths of two sides $l_{0}$ and $l_{3}$ (thick lines on the figure) is sufficient to determine a unique quadrilateral with internal angles $\alpha, \beta, \gamma, \delta$. The last point $C$ (white dot on the figure) is found by intersecting two lines (dashed lines on the figure). For the sake of simplicity, we consider planar quadrilaterals in the reference plane $(A B D)$ : the equations are written in a frame centred in $A$ and represented by the blue arrows in the figure. The intersection is found by solving the following equation:

$$
\left(\begin{array}{c}
l_{0}+l_{3} \cos \alpha \\
l_{3} \sin \alpha
\end{array}\right)=\left(\begin{array}{cc}
\cos \beta & \cos (\alpha-\delta) \\
-\sin \beta & \sin (\alpha-\delta)
\end{array}\right) \cdot\left(\begin{array}{l}
l_{1} \\
l_{2}
\end{array}\right)
$$
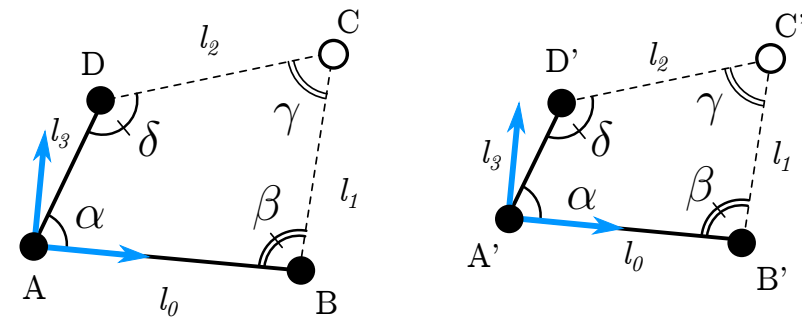

Figure 2: Two quads related by a Combescure transformation.

In the same way, prescribing the lengths of all edges on two intersecting lines, as shown in Figure 3 is sufficient to determine the entire parallel mesh. In this image, the thick lines correspond to edges which have prescribed lengths. Starting from a quadrilateral with two prescribed lengths, it is possible to apply equation (4) and find the last point of the quadrilateral (white dot). It is then possible to apply 

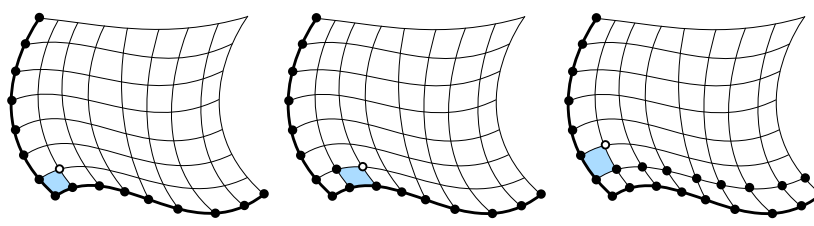

Figure 3: Propagation method for the computation of a Combescure224 transformation with quadrangles.

this procedure to the next quadrangle in the same row, and so forth, up to completion of each strip.

This iterative procedure is computationally efficient. ${ }_{227}$ The number of operations and the use of memory is pro- 228 portional to the number of faces in the mesh, as the solu-229 tion of the propagation requires $N M$ applications of equa-230 tion (4) for a mesh of $N$ times $M$ facets. The computation $_{23}$ time also varies linearly with the number of faces, as dis-232 cussed in [14]. This technique is thus more efficient $\operatorname{than}_{233}$ SVD, which requires assembling of matrices. The com- ${ }^{234}$ putational gain is especially important for large meshes $_{235}$ and makes the method proposed in this paper suited for $_{236}$ real-time applications.

\subsection{Super-canal surface}

We call super-canal surfaces the surfaces that are $^{239}$ images of canal surfaces by arbitrary compositions of ${ }^{240}$ Combescure transformations and Möbius transformations. ${ }_{241}$ This name recalls the term supercyclide to name projec- ${ }_{242}$ tive transforms of Dupin cyclides by Pratt [30]. We choose to use the same prefix even if the transformations at stake ${ }^{243}$ in this paper are different from the ones studied by Pratt. ${ }^{244}$

The image of a circular quad-mesh by Combescure ${ }^{245}$ transformations and inversions remains a circular quad- ${ }^{246}$ mesh, but both transformations affect differently the over- ${ }_{247}$ all shape, creating interesting formal possibilities. The $\mathrm{two}_{248}$ operations do not commute, so specifying the order of ap- 249 plication of Combescure and Möbius transformation has ${ }_{250}$ an influence on the properties of the final shape.

Hence, the methodology proposed in the following is to ${ }^{251}$ reconstruct a super-canal surface from two curves assum- ${ }^{252}$ ing a composition of applications of inversions or Combes- ${ }^{253}$ cure transforms. Rather than playing with canal surfaces and transformations, the principle of the method relies on a reverse approach which aims at finding an initially unknown canal surface that would satisfy two prescribed boundaries (see Section 5.1).

\section{Super-canal surfaces}

\subsection{A general framework for shape generation}

The method exposed above translates into a simple framework that requires two perpendicular curves as input. Indeed, canal surfaces do not have umbilical points (except poles), and consequently, their lines of curvature are necessary perpendicular. The designer thus chose a rule of construction for the surface, i.e. a specific combination of Combescure and Möbius transformations. The concatenation of transformations provides more design freedom to the end-user than the utilization of one specific transformation: this is discussed in the next sections. The identified families are proposed in Figure 4. The nomenclature for the different surfaces follows:

- the letter $C$ denotes that the initial shape was subjected to a Combescure transformation;

- the letter $M$ denotes that the initial shape was subjected to a Möbius transformation;

- the order of the letters gives the order of composition of the transformations: $C M$ means that the initial shape was subject to a Möbius transformation, then a Combescure transformation;

- the name of the initial shape subject to the transformations stands at the end: for example a $M$-revolution surface is an inversion of a surface of revolution.

Many surfaces well-identified in the literature can be generated with this method as illustrated in Figure 4. All the common surfaces used for geometrically-constrained 


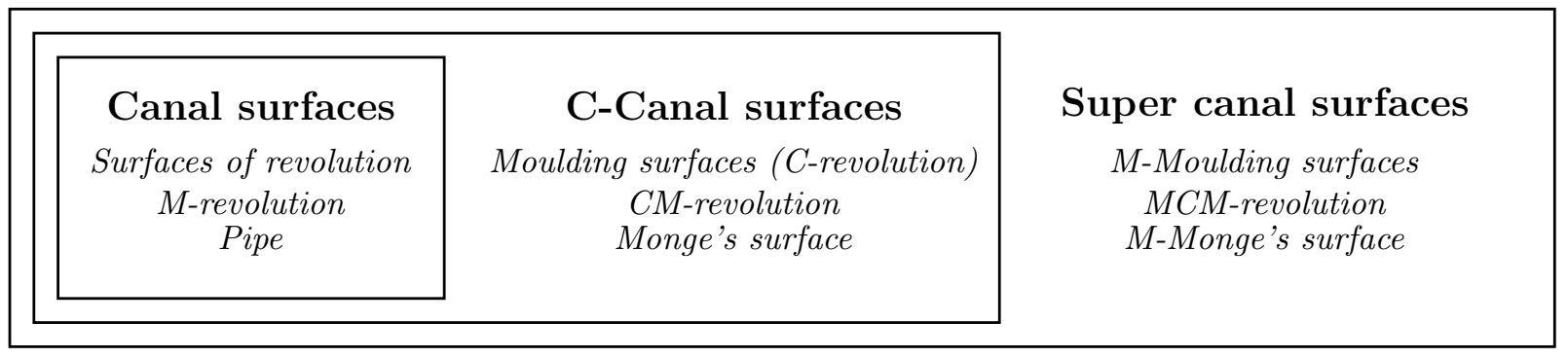

Figure 4: Super canal surfaces

methods mentioned in Section 1.2 fall into the category 281 of super-canal surfaces, with the exception of scale-trans surfaces. The curves used in surfaces of translation and scale-trans surfaces do not correspond in general to lines of curvatures and cannot be approached by circular meshes. Therefore, they do not have any specific offset properties.

It appears that moulding surfaces and Monge surfaces discussed in [17] are a subset of the shapes generated by Combescure transformations of canal surfaces. From a practical point of view, shapes with a family of planar curves are of great interest in construction. For that reason, we restrict the examples of application to $C M$-surfaces, where the families of circles are transformed into planar curves.

\subsection{Input for design with super-canal surfaces}

In the following of [3], we propose to design super-canal 284 surfaces from two curves. The simplest way to parame-285 terise a canal surface is to take a strip of circles as input286 parameters, as pictured in Figure 5. A two parameters ${ }_{287}$ family of cyclidic nets can be supported on the circular $_{288}$ mesh: the choice of those parameters can be done to fulfill ${ }_{289}$ some design requirements, like the shape smoothness, eval- ${ }_{290}$ uated with conformal Willmore energy [31, 22]. In the example of Figure 5, eight circles in the same plane are used to generate a canal surface. Only the portion of the canal surface above the construction plane is shown. Note also that the resulting surface, made of cyclidic patches, is a
$C^{1}$ surface with curvature discontinuities between patches.

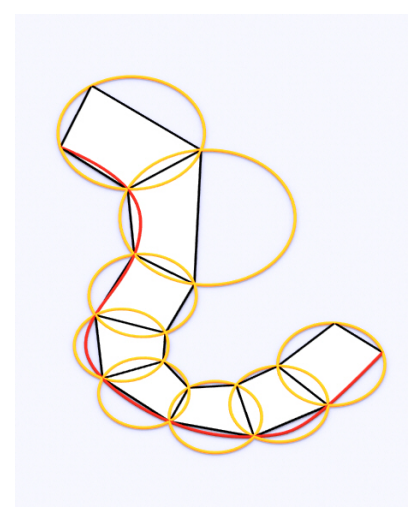
a given curve (red) (a) Circular strip supported on

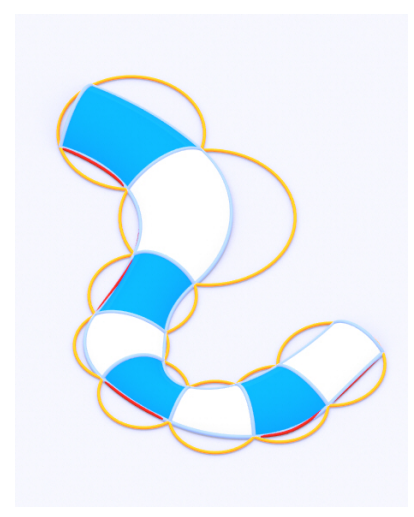

(b) One of the cyclidic nets supported on the circular strip
Figure 5: A canal surface created from a coarse circular strip

To define the strip of circles, the user can draw manually a collection of circles, or entirely parametrised it by a boundary curve and the radii of circles or a target length for each border. The latter parametrisation is depicted in Figure 6, whose input data follows:

1. a list of points on a curve in space;

2. one point $\mathbf{P}$ in space defining the first circle;

3. a function describing the lengths of each edge crossing the strip (thick orange lines on Figure 6).

It is then possible to construct one unique circular strip passing through the input points by propagation, in the manner of [32]. The construction of a circular strip restricts the two boundaries to be lines of curvature of the resulting surface. Section 4.1 will show how this condition 


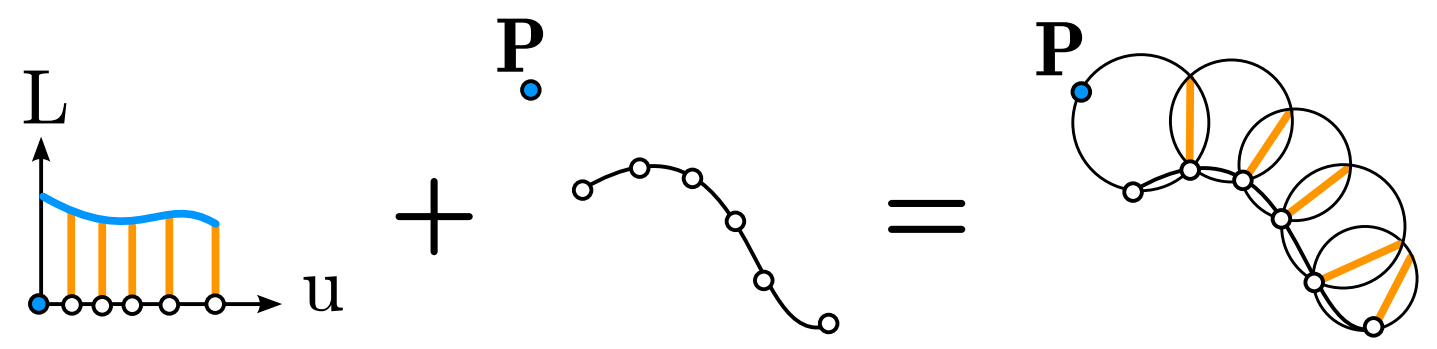

Figure 6: Parameters creating a circular strip that can support a canal surface

can be relaxed, while keeping the parametrisation of the 309 shapes by cyclidic nets and circular strips.

\subsection{Shape smoothing}

Some input data might lead to visually unpleaseant results, therefore we use the strategy proposed in [26] and take the position of the vertices and the orientation of the normal vector to the cyclidic net at one node as variables for smoothness optimisation. The objective is to fit exactly one input curve. To this end, the points on this curves are parametrised by the vector $\mathbf{u}$. The other parameters governing the shape of the canal surface are the lengths of the edges crossing the circular strip $\mathbf{L}$ (see Figure 6). The cyclidic net is then generated by the choice of an orthog- $^{-}{ }_{312}$ onal frame, parametrised by two angles $\lambda$ and $\theta$, which are angles defining a spherical coordinate system. The smoothness functional $\mathcal{F}$ is finally defined as a quadratic function of the radii of the edges of the cyclidic net $R_{\text {edge }}{ }_{316}$

$$
\mathcal{F}(\mathbf{u}, \mathbf{L}, \lambda, \theta)=\sum_{\text {edges }} \frac{1}{R_{\text {edge }}^{2}(\mathbf{u}, \mathbf{L}, \lambda, \theta)}
$$

The computation of the function is not hard, and its minimisation gives satifying results and is done in real-time. The user can specify additional constraints, like the angle made by the normal and a reference plane. In the latter ${ }^{321}$ case, the degrees of freedom $\lambda$ and $\theta$ become coupled, and ${ }^{322}$ the normal rotates along a cone.

Practically, the minimisation is here done by the means ${ }_{324}$ of the Broyden-Fletcher-Goldfarb-Shanno (BFGS) algo- $_{325}$ rithm. Figure 7 shows the smoothing of a canal surface ${ }_{326}$ based on the proposed energy. The parameters are the circle radii. Note that only local changes are introduced after optimisation, and that the areas where the facets were degenerated have disappeared.

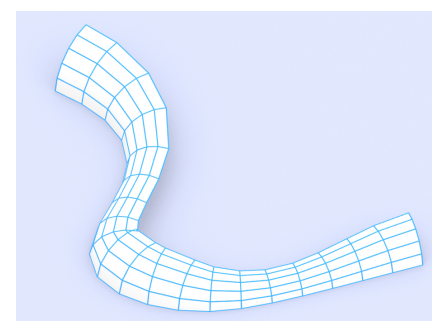

(a) Before optimisation

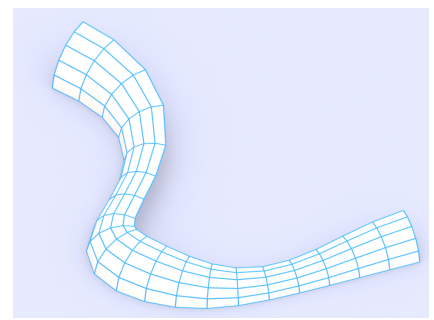

(b) After optimisation
Figure 7: Optimisation of the smoothness of a canal surface.

\subsection{Mechanical properties of super-canal surfaces}

This Section discusses briefly the mechanical behaviour of super-canal surfaces. C-canal surfaces play indeed a particular role in shell theory, as Rogers and Schief proved that their lines of curvatures are also lines of principal stresses under a uniform external load [21]. This result was also proven for canal surfaces before in [33].

This induces two remarkable features for the behaviour of the shapes previously presented:

- principal stresses lines following principal curvature lines, the natural mesh of C-canal surfaces is an optimal mechanical layout for a grid structure;

- all closed shapes generated by this method are in equilibrium under uniform pressure and therefore suited for pneumatic structures. 
Before showing the shape generation framework, we 363 should make a comment on potential applications for shal-364 low roof structures. A normal pressure load is surely very 365 close to a uniform distributed load for surfaces with mod-360 erate curvature. It can be concluded that shallow canal ${ }_{36}$ surfaces are close to funicular shapes under uniformly dis-368 tributed load. This kind of consideration has been docu-369 mented for shallow arches: shallow circular arcs, parabolazто or catenary have similar geometry and mechanical be-371 haviour, especially buckling capacity. For more comments 372 on this topic, the reader can refer to [34].

Furthermore, it should be recalled that in practice, 374 temporary actions are not negligible compared to the selfweight of a structure, and gridshells or thin shells are often designed with respect to non-symmetrical loads, for which lines of curvature are not principal stress lines. The finding of a structural optimum for different load cases combinations is far from obvious, but its computation is not necessarily a practical design objective: just like fabrication, structural performance is not the only criterion taken into account by the architects and engineers. The integration of principal stress under self-weight can be seen as a simple way to generate a good, but not necessarily optimal structural pattern, while creating a rich design narrative referring to pioneering works of structural artists. Lines of principal stress have been used by Pier Luigi Nervi for 375 the design of concrete ribbed slabs. Nervi did not solve ${ }_{376}$ an optimisation problem, but used a simple guiding prin- ${ }_{377}$ ciple for his design, which resulted indeed in highly efficient structures [35]. The meshing of super-canal surfaces 379 by their lines of curvatures combines thus constructability ${ }_{380}$ with structural efficiency.

\section{Application to shape modelling}

\subsection{Generation of canal surfaces}

The previous section discussed how canal surfaces can be parametrised with circular strips supporting cyclidic ${ }^{386}$ nets. This generation method leads however to a strong formal restriction, as it forces the two boundaries of the strip to be lines of curvature of the resulting canal surface. The practical consequence is that the second curve is restricted to be on a developable surface passing through the first curve, whereas the designer would prefer to define it independently. This section introduces thus an original algorithm for the shape generation of canal surfaces from two curves where only one of the two curves is a line of curvature of the canal surface. The problem is illustrated in Figure 8 and it will be shown that it admits a one parameter family of solutions.

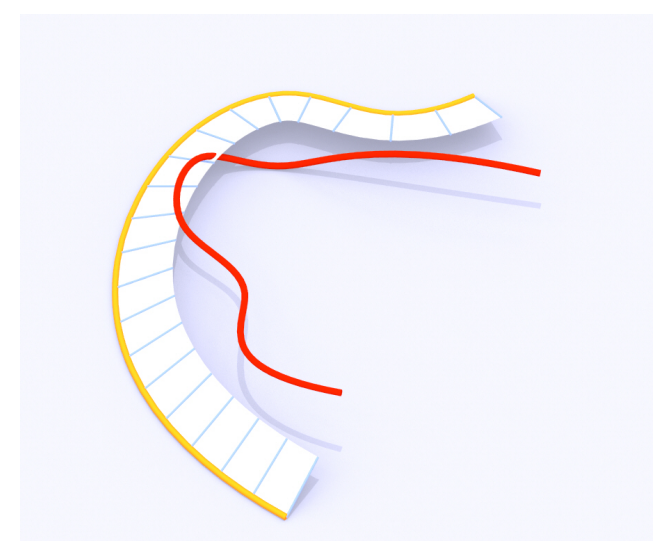

Figure 8: Input data for the curve-fitting problem. Line of curvature (orange), line to fit (red), and surface (white) containing the centers of the spheres.

\section{Preliminary considerations}

The relevant definition of canal surfaces in this case is to consider them as the envelope of a family of spheres. Remarkable properties of canal surfaces, and of lines of curvature in general can be mentioned:

1. Canal surfaces are envelopes of spheres, and as such, the spheres generating the surface meet tangentially with any curve of a canal surface.

2. The envelope of the lines directed by the normal of the surface along a line of curvature is a developable surface.

From remark 1, we get that the locus of the centers of the spheres generating the canal surface is on the surface 

resulting canal surface.

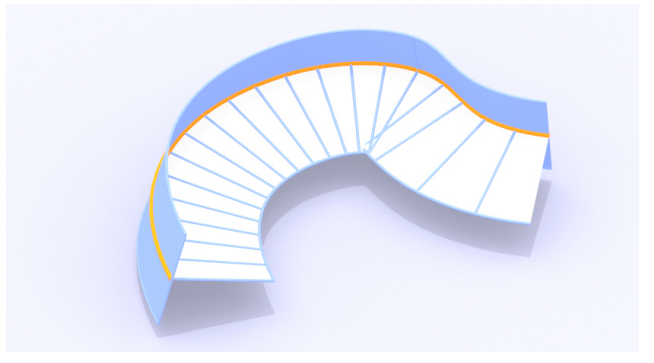

Figure 9: Line of curvature: one developable surface containing the centres of the spheres (white). The developable surface perpendicular to it (blue) is tangent to the resulting canal surface (not shown in the Figure).

generated by the normals of the surface. From remark 415 2 , we get immediately that this is a developable surface. Actually, it is a specific case of Monge surface [17]: once one normal has been chosen, the other normals are determined uniquely so that the envelope is indeed a developable surface. The locus of the centres of the spheres is therefore controlled by one orientation parameter. This is illustrated in Figure 9: choosing the orientation of the normal is equivalent to choosing a surface tangent to the

\section{Computation of the locus of centres}

Consider now that a normal vector and a line of curvature have been specified for the canal surface. The locus of centres is on a developable surface. So far we did not use any property of the second curve. We notice however that the centres of the spheres are on the bisector surface of the two curves. Such surface is defined as the envelope ${ }^{419}$ of the points which are equidistant to both curves. They ${ }^{420}$ have been studied in [36] for example.

Therefore, the centres of the spheres can be found by ${ }_{422}$ intersecting the bisector surface of the two curves and the 423 developable surface constructed from the normals. Both 424 surfaces are not bounded, and it seems intuitive that they ${ }_{425}$ will have an intersection in non-degenerate cases. The 426 construction of the whole bisector surface is however not 427 necessary, as it is meaningful to consider a finite collec-428 tion of spheres that will construct the cyclidic net that ${ }_{429}$ parametrise the canal surface.

Consider hence the first curve discretised with $n$ subdivisions, as depicted in Figure 10. The centres of the spheres belong to $n$ lines on the developable surface. Let $\mathbf{P}_{k}$ be the $k^{t h}$ point on the first curve, $\mathbf{C}_{k}$ the centre of the bi-tangent sphere on the corresponding line and $\mathbf{C}^{\prime}{ }_{k}$ the closest point to $\mathbf{C}_{k}$ on the second curve. By default, $\mathbf{C}_{\mathbf{k}}$ is not on the bisector surface. Therefore, the following functional is introduced and minimised.

$$
F=\sum_{k=0}^{n}\left(\left\|\mathbf{C}_{k} \mathbf{P}_{k}\right\|-\left\|\mathbf{C}_{k} \mathbf{C}_{k}^{\prime}\right\|\right)^{2}
$$

The positions of the $\mathbf{C}_{k}$ are encoded with independent unique parameters. Each term of the sum can thus be minimised individually by the means of Newton's method.

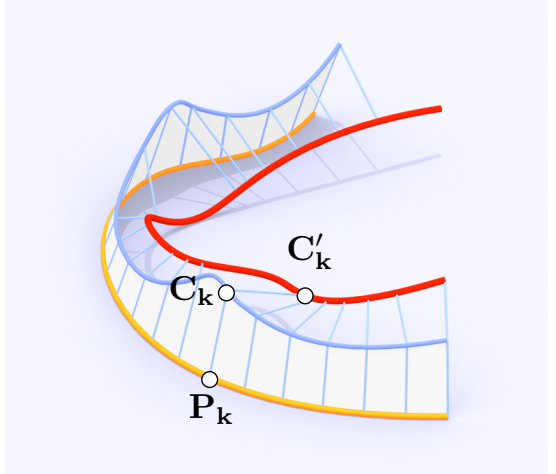

Figure 10: The curve fitting problem: the locus of the sphere centres (in dark blue) belongs to the developable surface chosen by the user, and each $\mathbf{C}_{\mathbf{k}}$ belongs to a straight line of this surface. The locus of centres is equidistant to both input curves.

\section{Algorithm for spheres generation}

The algorithm for the generation of a canal surface from two curves follows:

1. Select two curves, one of them being a line of curvature on the final surface.

2. Choose an orientation of the canal surface: specifying one orientation restricts the locus of centres to be in a uniquely defined developable surface.

3. Discretise the line of curvature with points $\mathbf{P}_{k}$, and generate the lines containing the centres of the spheres on the developable surface. 
4. Initialise the $\mathbf{C}_{k}$ with $\mathbf{C}_{k}=\mathbf{P}_{k}$.

5. Minimise Equation(6) with Newton's method.

The result is a collection of points corresponding to sphere centres. The radius $R_{k}$ of each sphere is given by the ${ }_{450}$ distance $\left\|\mathbf{C}_{k} \mathbf{P}_{k}\right\|$.

\section{Generation of a supporting cyclidic net}

We have seen that given two curves and a supplemen- ${ }^{45}$ tary condition, it is possible to define one unique family ${ }^{454}$ of spheres that optimally fits the two curves. Consider ${ }^{455}$ now the circles $\mathcal{C}_{k}$ defined as the intersection of successive ${ }^{456}$ spheres $\mathcal{S}_{k}, \mathcal{S}_{k+1}$, like shown in Figure $11 . \mathbf{P}_{\mathbf{k}}$ is the point ${ }^{457}$ of $\mathcal{C}_{k}$ on the input curve.

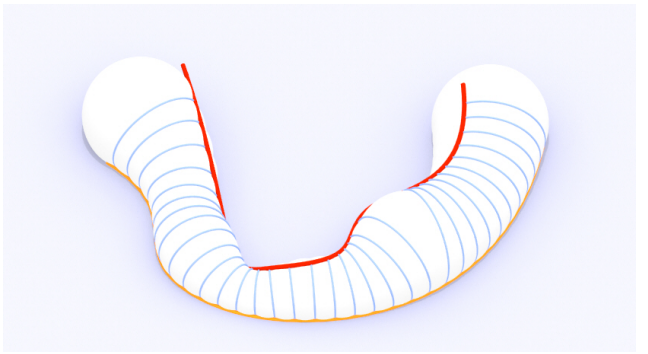

Figure 11: A family of spheres (white) fitting two curves (red and ${ }^{465}$ orange), and their successive intersection (blue).

It is clear that for any $k, \mathcal{C}_{k}$ and $\mathcal{C}_{k+1}$ both belong ${ }^{467}$ to the sphere $\mathcal{S}_{k+1}$. Consider Figure 12: choosing one ${ }^{468}$ point $\mathbf{V}_{k}$ on $C_{k}$ there is exactly one point $\mathbf{V}_{k+1}$ on $\mathcal{C}_{k+1}{ }^{469}$ so that $\mathbf{P}_{k} \mathbf{V}_{k} \mathbf{V}_{k+1} \mathbf{P}_{k+1}$ is inscribed within a circle. The $\mathrm{e}^{470}$

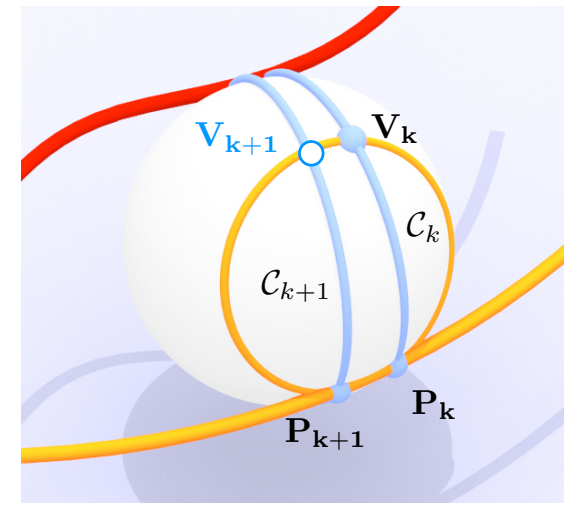

Figure 12: Two circles $\mathcal{C}_{k}$ and $\mathcal{C}_{k+1}$ : by choosing one point $\mathbf{V}_{\mathbf{k}}$ on $\mathcal{C}_{k}{ }^{478}$ one defines a circle and a one parameter family of cyclidic patches. ${ }^{479}$

\section{the}

46 .

.

(n)

strip, supporting a cyclidic net. The circles $\mathcal{C}_{k}$ can be edges of the resulting cyclidic strip because they belong to the same sphere [25].

\section{Comment}

The proposed method allows for the construction of a canal surface that fits optimally two input curves. The surface can be parametrised instantly with cyclidic patches and covered with a circular mesh as detailed in [25]. Consider indeed the collection of circles $\left(\mathcal{C}_{n}\right)$, and two consecutive circles $\mathcal{C}_{n}$ and $\mathcal{C}_{n+1}$. By construction, these circles are co-spherical, and thus, any planar quad with vertices on $\mathcal{C}_{n}$ and $\mathcal{C}_{n+1}$ is also a circular quad. This simplifies greatly the meshing process. The meshing algorithm is illustrated in Figure 13.

- Choose a discretisation of the first circle $\mathcal{C}_{1}$, the $\mathrm{k}^{\text {th }}$ point of the $n^{t h}$ circle is noted $\mathbf{P}_{\mathbf{n}, \mathbf{k}}$

- The $\mathbf{P}_{\mathbf{n}, \mathbf{1}}$ are chosen so that they all belong to the fitted curve which is a line of curvature of the resulting surface;

- Starting from $k=1$ and $n=1$, generate the plane $\mathcal{P}$ going through $\mathbf{P}_{\mathbf{n}, \mathbf{k}}, \mathbf{P}_{\mathbf{n}, \mathbf{k}+\mathbf{1}}$ and $\mathbf{P}_{\mathbf{n}+\mathbf{1}, \mathbf{k}}$ (step 1 in Figure 13);

- The point $\mathbf{P}_{\mathbf{n}+\mathbf{1}, \mathbf{k}}$ is the intersection between $\mathcal{P}$ and $\mathcal{C}_{n+1}$ (step 2 in Figure 13);

- Iterate over $k$ (step 3 in Figure 13);

- Iterate on $\mathcal{C}_{n+1}$ and $\mathcal{C}_{n+2}$ (step 4 in Figure 13);

The tool recalls the two-rails sweep commonly used in CAD software. One curve is a line of curvature of the resulting shape. It provides proper alignment of the mesh with the borders, which often dictate the mechanical behaviour of the structure.

\subsection{Generation of closed canal surfaces}

The proposed construction can be extended to closed 480 strips with several limitations. The first one has been discussed in [22]: a closed cyclidic net gives a smooth closed 

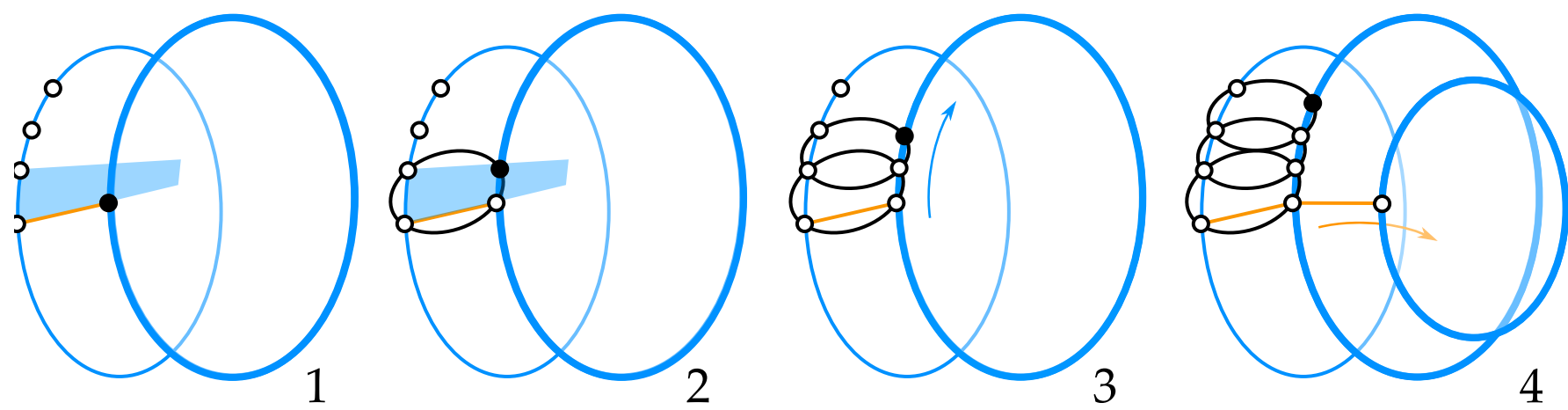

Figure 13: Meshing between two co-spherical circles (axonometry).

surface if and only if the discrete guide curve is a pseudospherical curve (it is parallel to a curve which has all its vertices inscribed within a sphere). The second condition corresponds to the possibility of drawing the last circle of the strip. Consider Figure 14: the first circle of the strip is written $C_{0}$, the penultimate circle $C_{f}$, the initial point $\mathbf{P}$ and the first and last point of the curve $\mathbf{P}_{\mathbf{0}}$ and $\mathbf{P}_{\mathbf{f}}$ respectively. There are two cases:

- $C_{0}$ and $C_{f}$ belong to the same sphere, then the circle going through $\mathbf{P}, \mathbf{P}_{\mathbf{0}}$ and $\mathbf{P}_{\mathbf{f}}$ intersects the circle $C_{f}$ in two points. This circle is the solution we are looking for and is represented with dashed lines on Figure 14.

- In the other cases, the spheres $\left(C_{0}, \mathbf{P}_{\mathbf{f}}\right)$ and $\left(C_{f}, \mathbf{P}_{\mathbf{0}}\right)$ are distinct. Their intersection is a circle intersecting ${ }^{511}$ the circle $C_{0}$ and $C_{f}$ in two different points. This ${ }^{512}$ circle is the only solution that allows the closing of ${ }^{513}$ the circular strip, and it does not intersect $C_{0}$ in $\mathbf{P} .{ }^{514}$

In the first case, only the intersection of the last circle and $C_{f}$ is unknown. In the second case, the position of $\mathbf{P}_{517}$ cannot be specified arbitrarily (as in section 2. for open strips). Compared to open strips, there is therefore a loss of at most two degrees of freedom for the control of the shape.

Figure 15 shows a rendering of a facade covered with a canal surface (the structural system supporting the cantilevering facade is not shown). Being able to model closed

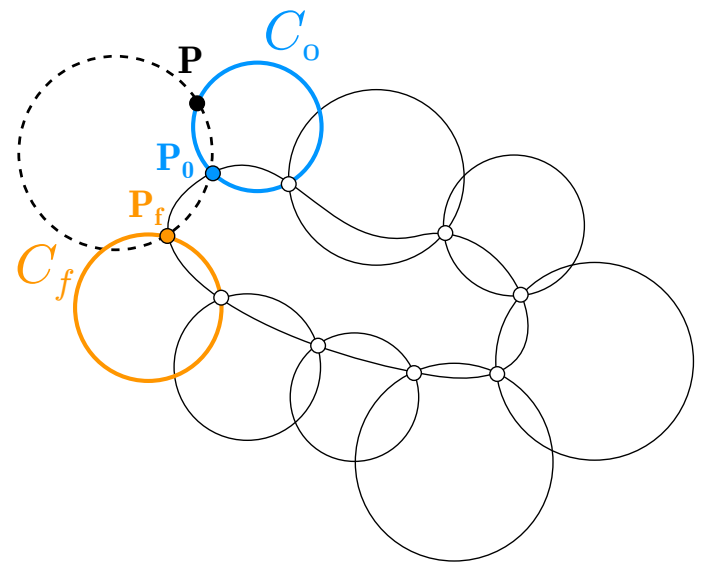

Figure 14: Problem of a closing strip

surfaces is crucial for architectural shapes, as façades are usually closed.

\subsection{Practical applications}

The method presented in this paper has been used during a one week workshop in 2015. Architecture and engineering students had to design and build a $30 \mathrm{~m}^{2}$ free-form pavilion, the only material available was polystyrene in flat rectangular sheets. The shape is a super-canal surface meshed with circular quadrilaterals. The pavilion, shown in Figure 16 is a grid structure with a torsion-free beam layout. The offset was computed with a reflection rule similar to the one generating cyclidic nets. An optimisation was performed in order to minimise the height gap at the nodes between beams of constant height. The fast computation of the space of solutions was key to the success of 


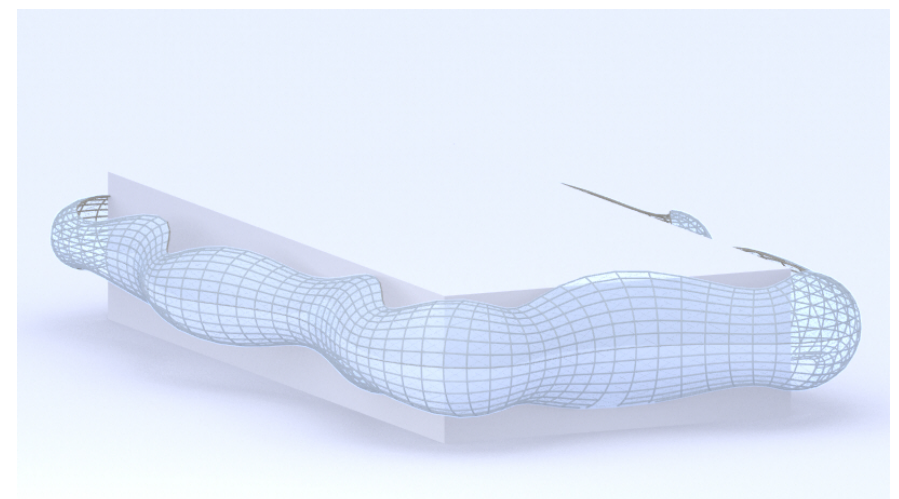

536

Figure 15: A visualisation of a façade as a canal surface, covered $^{537}$ with a circular mesh.

this operation within a limited time frame (5 days).

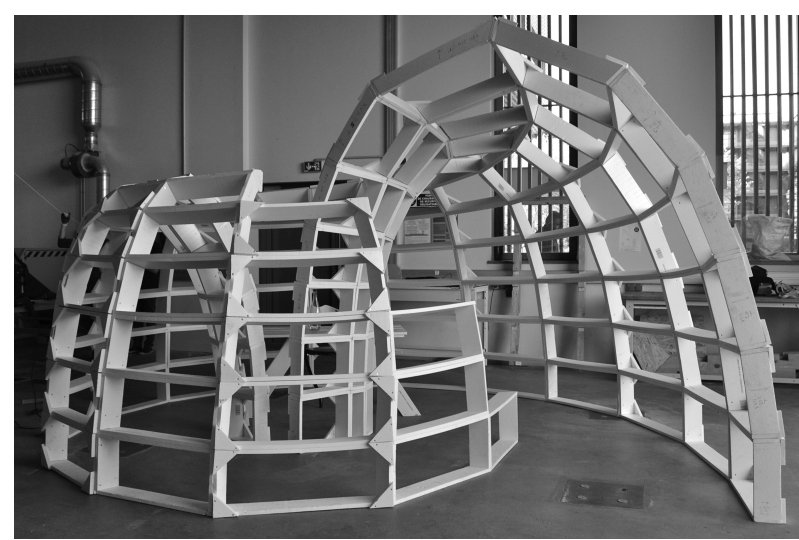

Figure 16: A prototype built with torsion free-nodes on a super-canal ${ }_{540}$ surface. The tools presented in this paper were used for shape ${ }^{542}$ generation as well as fabrication. Hundreds of polystyrene ${ }^{543}$ elements were cut according to the $3 \mathrm{D}$ model and assem- ${ }^{544}$ bled. The planarity of the panels was considered for use ${ }^{545}$ as bracing elements and was validated on a $5 \mathrm{~m}^{2}$ model, ${ }^{546}$ shown in Figure 17. Flat panels used as bracing elements ${ }^{547}$

Another exploration was performed with a timber structure, shown in Figure18. The structure is a plated ${ }^{549}$ shell structures: the facets are connected along their edges, ${ }_{550}$ without additional stiffeners. The small-scale pavilion il-
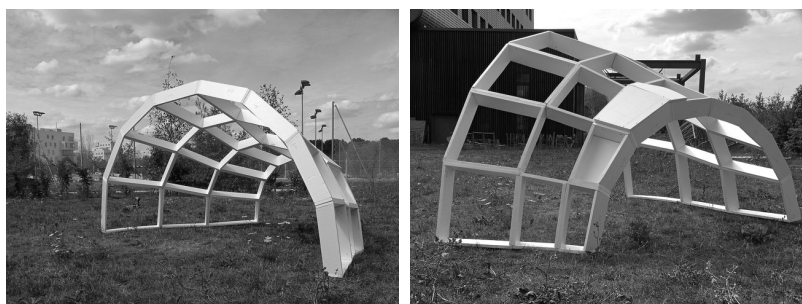

Figure 17: A model of a canal surface with planar quadrangles used for bracing.

lustrates thus the potential offered by planar panels rather than offset properties of circular meshes, although the discrete normals of circular meshes have been used to generate planar cuts between the plates.

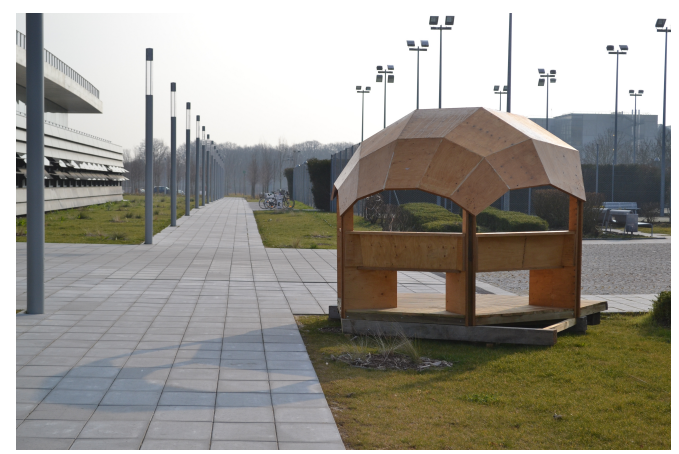

Figure 18: A timber plated shell structure covered with circular quadrilaterals generated with the method proposed in this paper.

The construction of those prototypes validates the use$$
542
$$
improve the overall stability and stiffness ${ }^{1}$.

\footnotetext{
${ }^{1}$ More details and pictures can be found on ${ }^{552}$ http://www.thinkshell.fr/building-freeform-2015/.
}

\author{
(1)
} of the numerical tools presented in this paper. The user feedback allowed us to identify the most relevant way to model super-canal surfaces. In particular, the students found important to control at least one boundary curve. This explains why the method of generation of canal surfaces presented in this work focuses on the prescription of a boundary curve, and not on the curve supporting the centers of the sphere for example.

\section{Application to inverse problems}

\subsection{Generation of M-revolution surfaces}

The most well-known canal surfaces are surfaces of revolution. They indeed correspond to the case of a straight 
generatrix. Surfaces of revolutions have many interesting properties for applications in architecture. They are isothermic surfaces, which means that they can be discretised as Edge-Offset Meshes. Yet, isothermic surfaces are preserved by Combescure and Möbius transformations and they thus inherit this property.

In particular, we discuss here of a particular subset of 'super-surfaces of revolutions', where the center of inversion and the axis of revolution are in a horizontal plane, as shown in Figure 19. It is clear that the parallels of the surface of revolution are vertical in this case. Since inversions preserve circles and angles, we can deduce that this family of curvature lines remain vertical after inversion. Combescure transformations preserve planarity: applying another Combescure transformation yields a surface with591 planar arches. This additional property is particularly in-592 teresting for applications to structural system with contin-593 uous arches and secondary structure. A specific method594 has therefore been developed to generate these surfaces. It consists of solving the inverse problem detailed in the following.

The input data for the problem are displayed in Figure 19. The user prescribes one planar curve, one circle in the same plane comprising the ends $\mathbf{P}_{\mathbf{1}}$ and $\mathbf{P}_{\mathbf{4}}$ of the curve, and two points $\mathbf{P}_{\mathbf{2}}$ and $\mathbf{P}_{\mathbf{3}}$ on this circle. The objective is here to reconstruct the initial surface of revolution, therefore the problem is to find a center of inversion $\mathbf{C}$ so that the image of the quadrangle $P_{1} P_{2} P_{3} P_{4}$ is an isosceles trapezoid.

Isosceles trapezoids are the only cyclic quadrilaterals that have parallel opposite edges. Notice that the problem is planar and can thus be formulated with complex numbers. The parallelism corresponds to the fact the direction vectors are co-linear (identical up to a scaling by a real number $t$ ). Assigning the complex numbers $z_{1}, z_{2}{ }_{593}$ $z_{3}$ and $z_{4}$ to the points $\mathbf{P}_{\mathbf{1}}, \mathbf{P}_{\mathbf{2}}, \mathbf{P}_{\mathbf{3}}$ and $\mathbf{P}_{\mathbf{4}}$, and writing $z_{j, C}$ the complex number associated to the image of $z_{j}$ by an inversion of center $\mathbf{C}$, we obtain equation (7):

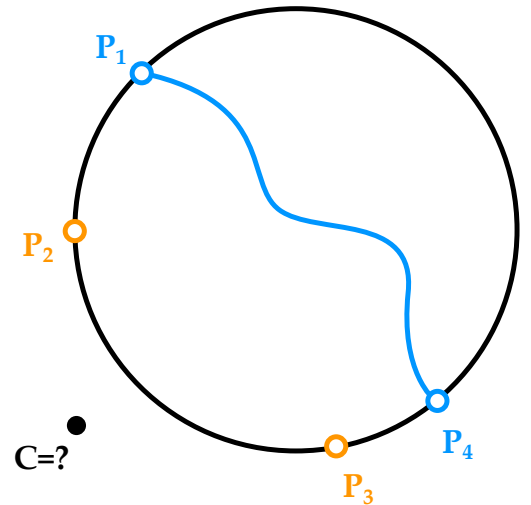

Figure 19: Problem for the practical design with inversion of surfaces of revolutions.

$$
\frac{z_{2, C}-z_{1, C}}{z_{3, C}-z_{4, C}}=t \in \mathbb{R}
$$

We can use the equation (2) to express equation (7) with respect to the $z_{j}$ and obtain equation (8). It is independent of the ratio of inversion $k$ : the position of the center of inversion is the only value of interest in this problem.

$$
\frac{\left(\frac{z_{2}-z_{1}}{\left(z_{1}-z_{C}\right)\left(z_{2}-z_{C}\right)}\right)}{\left(\frac{z_{3}-z_{4}}{\left(z_{3}-z_{C}\right)\left(z_{4}-z_{C}\right)}\right)}=t \in \mathbb{R}
$$

After simplifications, this equation leads to a second order equation in $z_{C}$. The general form of (8) can be written as:

$$
A_{t} z_{C}^{2}+B_{t} z_{C}+D_{t}=0
$$

with

$$
\left\{\begin{array}{ccc}
A_{t}= & z_{2}-z_{1}+t \cdot\left(z_{4}-z_{3}\right) \\
B_{t}= & -(1+t) z_{1} z_{3}+(t-1) z_{1} z_{4} \\
& & +(1+t) z_{2} z_{4}+(1-t) z_{2} z_{3} \\
D_{t}= & z_{3} z_{4}\left(z_{2}-z_{1}\right)+t z_{1} z_{2}\left(z_{4}-z_{3}\right)
\end{array}\right.
$$

The case of $A_{t}=0$ can occur only when the quad $P_{1} P_{2} P_{3} P_{4}$ is already an isosceles trapezoid. In the other cases, for each value of $t$, there are two complex solutions giving two positions for the center of inversion in the complex plane. It is thus possible to solve this inverse problem with a straight-forward solution based on complex analysis. 
An illustration of this problem is shown on Figure 20.628 On this image, all the facets are inscribed within circles.629 The free-form shape is thus covered with planar facets and torsion-free nodes. Since the circle shown in Figure 20 is in the horizontal plane, it is noticed that one family of lines of curvature consists of planar vertical arches. The solution proposed here can easily be extended to the case of a spherical guide curve with two successive inversions. Likewise, it is possible to apply this method to moulding or Monge surfaces.

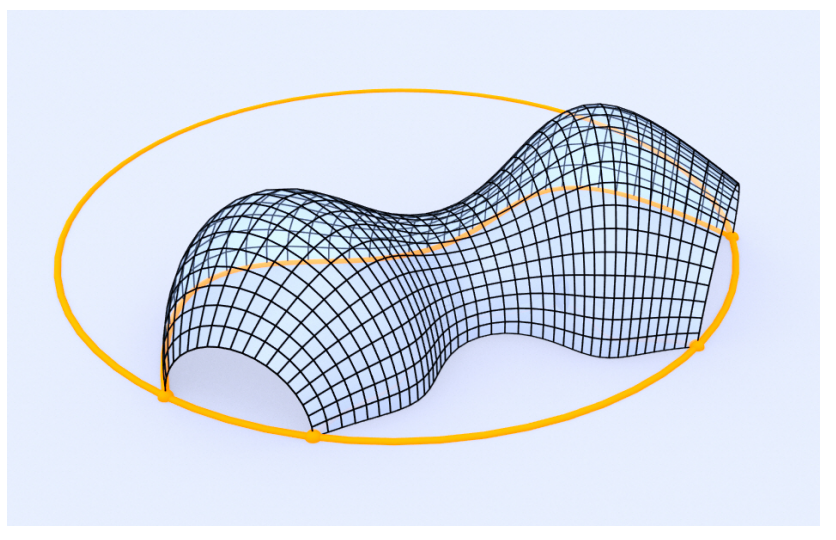

Figure 20: Surface generated by inversion of a surface of revolution ${ }_{633}$ constructed from one curve and two points on a circle.

\subsection{C-canal surfaces}

We proposed an extension of the generation method proposed in section 4.1 by adding a Combescure trans- ${ }^{63}$ form so that the can be any planar curve, and that the ${ }^{638}$ final surface is a C-canal surface. Figure 21a shows the ${ }^{639}$ three input data for the generation of a C-canal surface, ${ }^{640}$ while Figures $21 \mathrm{~b}$ and 21c show two possible outputs. Like ${ }^{641}$ canal surfaces, the user can specify one curve, a collection ${ }^{642}$ of lengths defining indirectly a second curve, and a planar ${ }^{643}$ cross-section that is obtained by Combescure transforma ${ }^{-644}$ tion of a circle. The inputs controlled by the designer $\operatorname{are}^{645}$ thus the same as the ones described in Figure 6, with the ${ }^{646}$ control of one curve in addition.

The lengths of the edges is specified for the C-canal ${ }^{648}$ surface, but at the beginning, only the canal surface $\operatorname{can}^{649}$ be computed. An optimisation procedure is thus required ${ }^{650}$ to find the canal surface that will fit the input data after Combescure transformation.

Writing $\mathbf{L}$ the target lengths for the curves crossing the C-canal surface (see Figure 6), we generate first the canal surface $\mathbf{F}(\mathbf{u}, \mathbf{L})$. There is one Combescure transformation $f$ that maps the first circle of the canal surface to the transverse input curves chosen while preserving one input curve. After the Combescure transformation, the resulting lengths $\mathbf{L}^{\prime}$ on the C-canal surface differ from $\mathbf{L}$. However, Figure 21 shows that a canal surface and a C-canal surface related by a Combescure transformation have similar boundaries, even if they do not perfectly coincide. Therefore, a local optimization algorithm (in our case BFGS) can be used to minimise the error:

$$
E\left(L_{k}\right)=\sum_{k}\left(L_{k}^{\prime}-L_{k}\right)^{2}
$$

The optimisation is done for each $L_{k}$ successively. This prevents from computing the whole Combescure transformation at each iteration, but only the strip where the error is evaluated. With this precaution, the computation remains lightweight and stable. This optimisation procedure can be extended to the fitting of two curves, like done in 4.1 .

\subsection{Meshing of super-canal surfaces}

A key feature of the proposed method is that it operates fundamentally on smooth surfaces. It is therefore independent from the mesh density. Notice for example that the solution of equation (9) does not require any knowledge on the discretisation of the curves, but only the four prescribed points. Therefore, re-meshing of super-canal surfaces is extremely simple and detailed below.

It has already been pointed out that inversions are involutions. Combescure transformations are linear maps and can easily be inverted with the algorithm proposed in Section 2.4. The computation of inverse transformation is thus extremely light. These properties are used extensively to remesh super-canal surface and is illustrated in 


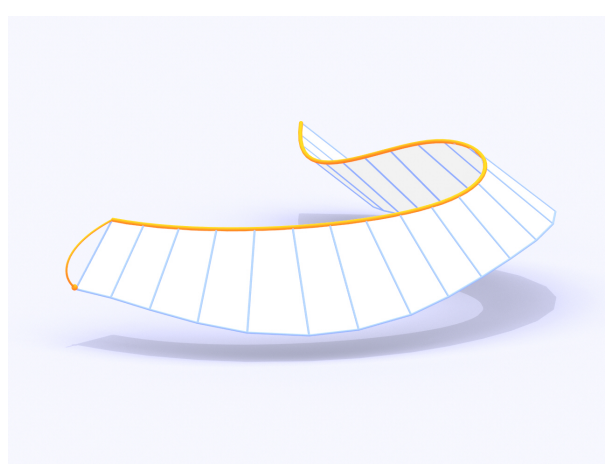

(a) Circular strip

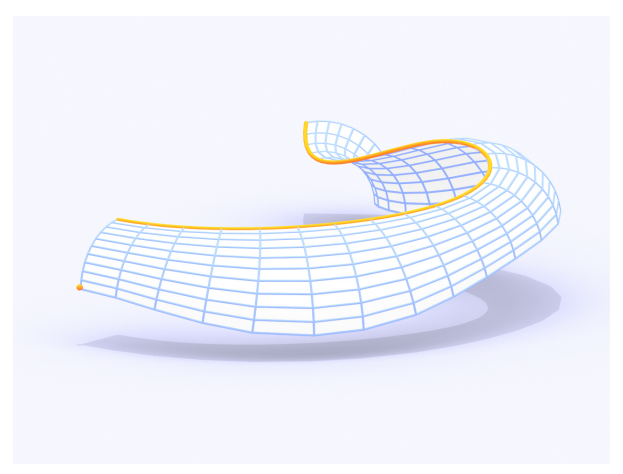

(b) A canal surface supported on the strip

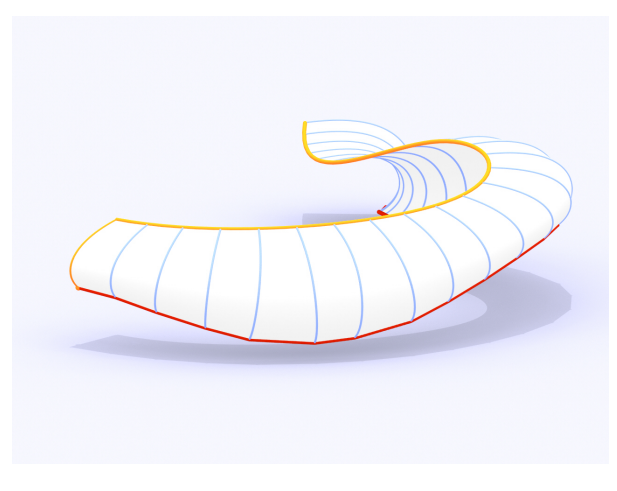

(c) A C-canal surface covered with D-strips

Figure 21: Generation of a C-canal surface

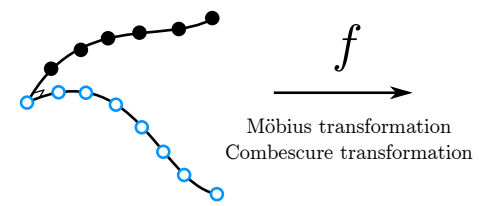

Reference curves

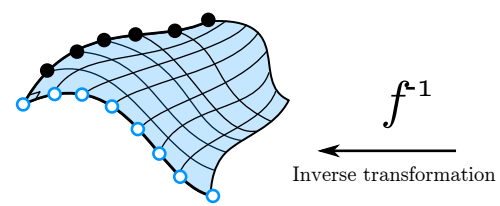

Final surface

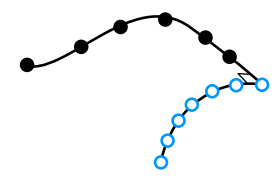

Boundaries of canal surface 673

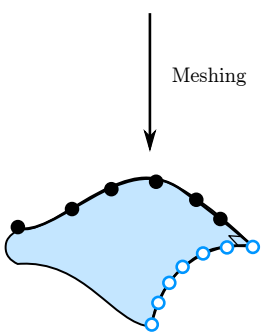

Figure 22: Remeshing procedure for a super-canal surface.

\section{Discussion}

\subsection{Algorithmic performance}

The algorithms of shape generation have been im- $_{686}$ plemented in Grasshopper ${ }^{\mathrm{TM}}$, an environment of visual ${ }_{687}$ programming compatible with the modelling software Rhinoceros ${ }^{\mathrm{TM}}$. In this section, we discuss the performance of the three operations used in our method:

- the computation of circular strips and the meshing of discrete canal surfaces;

- the computation of Möbius transformations and the solution of the inverse problems;

- the computation of Combescure transformations.

Circular strips are defined using a propagation algorithm. The problem solved at each step is the intersection of a sphere and a circle. The resulting computation time varies linearly with the number of subdivisions of the guide curve.

The computation of Möbius transformations is straight forward, as equation (1) is applied to each point of the mesh. Likewise the solution of equation (9) is obvious and requires no special numerical treatment. For that reason, Möbius transformations of meshes are as fast as the computation of simple affine transformations, like scaling or translation.

A case-study for the computation of Combescure transformations was performed. Like discussed in Section 2.4, the computation time varies linearly with the number of panels in the structure. The computation time is inferior to 20 milliseconds for a mesh with 10,000 faces, a high 


\begin{tabular}{|c|c|c|c|c|c|}
\hline & \multirow{3}{*}{ Isothermic } & \multirow[b]{2}{*}{ Planar curves } & \multirow[b]{2}{*}{ Stress lines } & \multirow[b]{2}{*}{ Circular mesh } \\
\hline & & & & & \\
\hline \multirow{3}{*}{ Canal surface } & revolution & & \multirow{6}{*}{ Yes } & \multirow{6}{*}{ Yes } & \multirow{9}{*}{ Yes } \\
\hline & M-revolution & Yes & & & \\
\hline & General case & No & & & \\
\hline \multirow{3}{*}{ C-canal surface } & C-revolution (moulding) & \multirow{2}{*}{ Yes } & & & \\
\hline & CM-revolution & & & & \\
\hline & General case & No & & & \\
\hline \multirow{3}{*}{ MC-canal surface } & M-moulding & \multirow{2}{*}{ Yes } & \multirow{3}{*}{ No } & \multirow{3}{*}{ No } & \\
\hline & MCM-revolution & & & & \\
\hline & General case & No & & & \\
\hline
\end{tabular}

Table 2: Properties of super-canal surfaces.

n that no pre-factorisation is required for the computation ${ }_{714}$ of the Combescure transformation: the computation time are the one experienced by the user. This is in accordance 716 with the will to offer a maximal flexibility for the design.717 Applying successive Combescure transformations is there-718 fore possible in real-time applications with our algorithm.719

Finally, we notice that Combescure transformations re-720 quire more time than the other operations and generally 721 governs the overall performance of the method. Notice also722 that Combescure transformations and Möbius transforma-723 tions have to be applied twice in the reverse engineering methods presented in the previous section.

\subsection{Properties of the structural layout}

Table 2 sums up the different properties of the surfaces created with our framework. As one applies Möbius and Combescure transformations and extends the formal freedom, some properties are lost a priori. All the shapes can nevertheless be parametrised as circular meshes. As an example, it can be noticed that the lines of curvature are not necessarily lines of principal stresses under uniform pressure for MC-canal surfaces. In the most general case, there is also no guaranty that there is a family of planar curves.

Among other remarkable properties, it may be noticed that the images of surfaces of revolution are isothermic surfaces, so that it is possible to parametrise them with conformal squares. Optimisation of the parametrisation of isothermic surfaces towards visually pleasant meshes could thus be done in the manner of [37].

Figure 23 shows a M-moulding surface. It was generated to fit two input curves, in the manner of M-revolution surfaces. The shape is visually not different from C-canal surfaces, but the curves are not planar, which increases the complexity. The analysis of Table 2 shows that C-canal surfaces are probably the best trade-off between design freedom and properties of the structural layout.

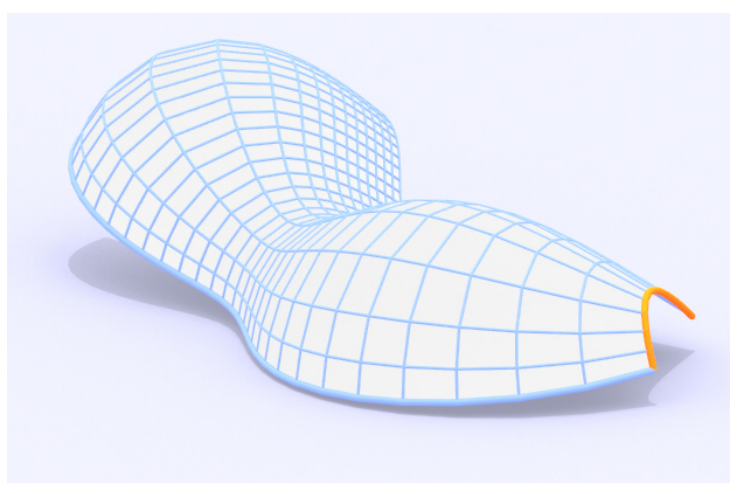

Figure 23: A M-moulding surface passing through two prescribed curves (thick lines). Only the orange curve is rigorously planar. 

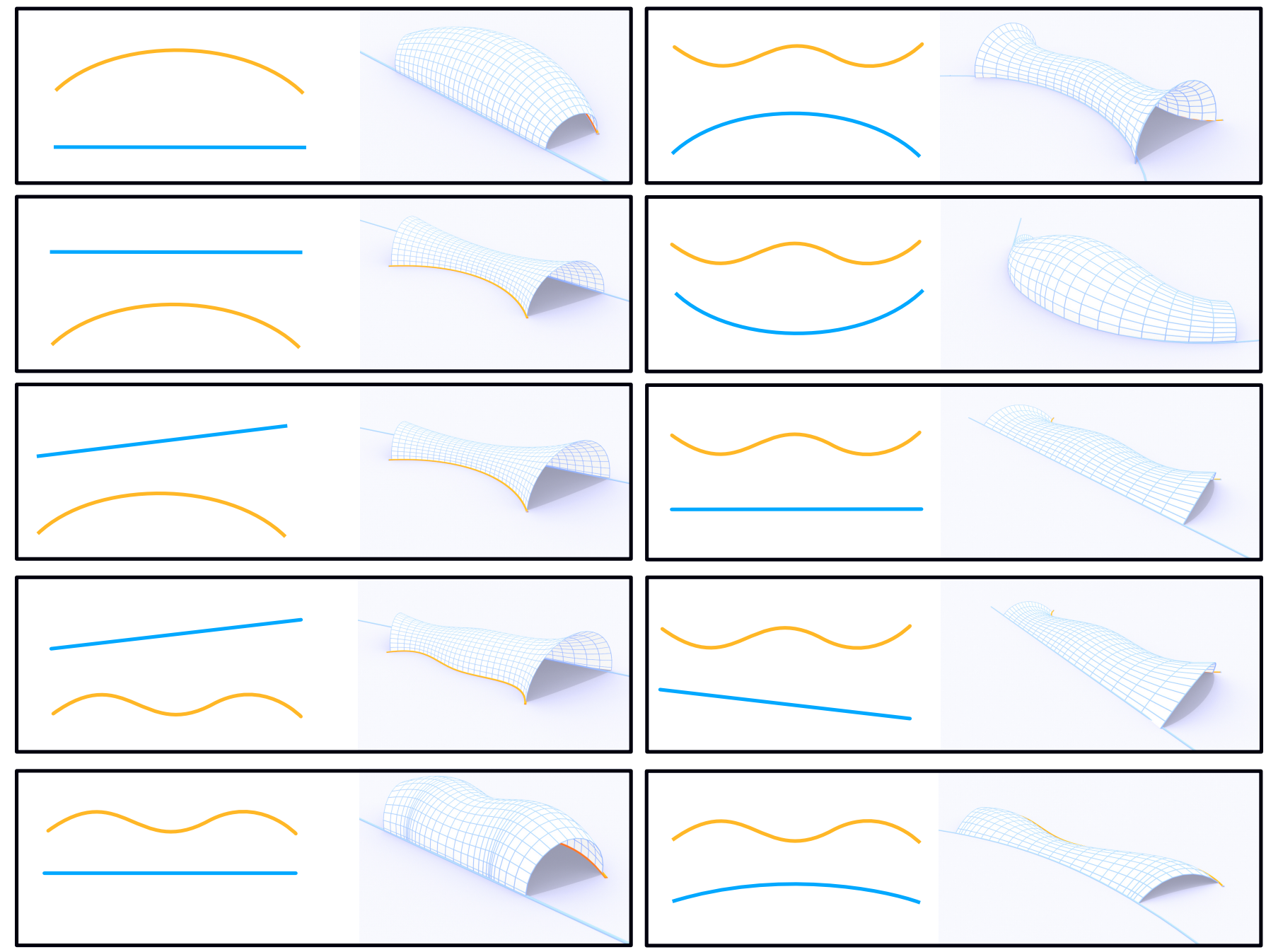

Figure 24: Canal surfaces obtained by fitting two planar curves, the orange curve is a line of curvature of the surface.

\subsection{Shape explorations: potential and limitations}

The generation super-canal surfaces is subject to modelling limitations discussed in this section. Figure 24 displays some canal surfaces generated by solving the twocurves fitting problem. The orange curve is a line of curvature of the resulting surface. The examples show that it is possible to properly fit a line with a doubly-curved shape, which should be of interest for practical applications.

These examples also highlight some limitations of ${ }^{739}$ super-canal surfaces. Consider for example the shape in ${ }^{740}$ Figure 25, where there is a noticeable shrinkage of panels. ${ }^{74}$ This concentration of lines of curvature is linked to the ${ }^{742}$ properties of the evolute of the guiding curve.

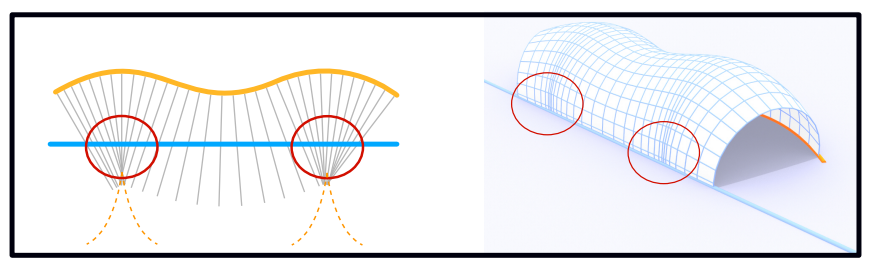

Figure 25: Concentration of lines of curvatures (circled in red) and plane view

The evolute of a curve is the locus of the center of its osculating circles. The lines obtained by sweeping along a curve intersect along the evolute, as shown in Figure 25. In Figure 25, it appears that the second curve used as an input for the fitting problem (in blue) is close to the evolute 
of the first curve (in orange). As a consequence, the planes781 containing the circles used for the shape generation (the ${ }_{782}$ grey lines in the top view) converge towards the evolute ${ }_{83}$ and result in panel shrinkage. This practical limitation ${ }_{784}$ also exists for more general super-canal surfaces. The de-785 signer must limit the curvature of the control curves in 786 order to avoid self-intersections of the surface he or she is787 generating.

\section{Conclusion}

This paper presented a new family of shapes for the ra- $^{791}$ tionalization of free-form structures and envelopes. It en- ${ }^{792}$ riches the formal vocabulary of geometrically-constrained design approaches, and has many relevant applications, ${ }^{793}$ from pneumatic structures to gelzed gridshells. A connec- ${ }_{794}$ tion to shell theory was recalled and showed that the sur- ${ }_{795}$ faces created with this method are at equilibrium under ${ }_{796}$ normal uniform load. Moreover, the lines of curvatures, ${ }_{797}$ that are used for discretisation of super-canal surfaces cor- $^{-}{ }_{798}$ respond to lines of principal stresses, making the meshes $_{799}$ efficient for both fabrication and structural performance. The tools developed for the shape generation were used $\mathrm{in}_{800}$ the practical context of a workshop for architecture and engineering students.

The methodology for shape generation relies heavily $y_{803}$ on Möbius geometry, the geometry of circles in space. ${ }^{804}$ It studies the transformations of shapes by Combescure ${ }^{805}$ and Möbius transformations, and in that sense, it is $\mathrm{a}_{807}$ generalisation of Möbius geometry, which is only inter-808 ested in the latter one. Like many other geometrically- ${ }^{809}$ constrained shapes, super-canal surfaces are generated ${ }^{810}$ from three curves. The underlying construction rule is si12 $_{12}$ more sophisticated than simple affine transformation, likes13 translation or scaling. It also gives more degrees of free- ${ }^{814}$ dom than scale-trans surfaces. Lack of design tools for ${ }^{815}$ designers and architects for complex structures has been ${ }_{817}$ shown by William Baker in a plenary talk at the Sym-818 posium of the IASS in 2015 , which is a real prejudice to the construction industry. Super-canal surfaces transcribe complex geometrical notions into tools easily usable as a design tool by architects and engineers as they provide insight on the buildability, the mechanical behavior under normal load of free-form structures. This illustrates the interest of using Möbius geometry for geometrical modelling in architecture. Other families of shapes could arise form this framework.

The shapes proposed in this paper could be combined with more general modelling techniques, for example see [38], who studies deformations of circular meshes by combination of compatible Möbius transformations.

\section{Acknowledgment}

This work was made during Mr. Mesnil doctorate within the framework of an industrial agreement for training through research (CIFRE number 2013/1266) jointly financed by the company Bouygues Construction SA, and the National Association for Research and Technology (ANRT) of France.

\section{References}

[1] J. Sakarovitch, Gaspard Monge founder of constructive geometry, in: Proceedings of the Third International Congress on Construction History, 2009, pp. 1293-1300 [cited May 2nd, 2016].

[2] J. Schlaich, H. Schober, Glass Roof for the Hippo Zoo at Berlin, Structural Engineering International 7 (4) (1997) 252-254. doi : https://doi.org/10.2749/101686697780494581.

[3] J. Glymph, D. Shelden, C. Ceccato, J. Mussel, H. Schober, A parametric strategy for free-form glass structures using quadrilateral planar facets, Automation in Construction 13 (2) (2004) 187-202. doi:https://doi.org/10.1016/j.autcon. 2003.09 .008 .

[4] Y. Liu, W. Wang, H. Pottmann, J. Wallner, Y. Yong-Liang, Geometric Modeling with Conical Meshes and Developable Surfaces, ACM Transactions on Graphics 25 (3) (2006) 681--689. doi:https://doi.org/10.1145/1179352.1141941. 
[5] J. Blassel, A. Pfadler, La gare de Strasbourg, Construction867 métallique 45 (1) (2008) 15-36.

[6] H. Pottmann, A. Schiftner, P. Bo, H. Schmiedhofer, W. Wang, 869 N. Baldassini, J. Wallner, Freeform surfaces from single curved870 panels, ACM Transactions on Graphics 27 (3) (2008) 1.871 doi:https://doi.org/10.1145/1399504.1360675]

[7] S. Stephan, K. Knebel, J. Sanchez-Alvarez, Reticulated Struc-874 tures On Free-Form Surfaces, Stahlbau 73 (2004) 562-572.875 doi:https://doi.org/10.1002/stab. 200490149.

[8] H. Pottmann, Y. Liu, J. Wallner, A. Bobenko, W. Wang,877 Geometry of multi-layer freeform structures for architec-878 ture, ACM Transactions on Graphics 26 (3) (2007) 65.879 doi:https://doi.org/10.1145/1276377.1276458.

[9] P. Bo, H. Pottmann, M. Kilian, W. Wang, J. Wallner, Circulars82 Arc Structures, ACM Transactions on Graphics (TOG) 30 (4)883 (2011) 101. doi:https://doi.org/10.1145/1964921.1964996. 884

[10] Y.-l. Yang, Y.-J. Yang, H. Pottmann, N. J. Mitra, Shapes8s Space Exploration of Constrained Meshes, ACM Transactions886 on Graphics 30 (2011) 124. doi:10.1145/2024156.2024158. 887

[11] S. Bouaziz, M. Deuss, Y. Schwartzburg, T. Weise, M. Pauly,888 Shape-Up: Shaping Discrete Geometry with Projections, Com-889 puter Graphics Forum 31 (5) (2012) 1657-1667. doi:10.1111/890 j.1467-8659.2012.03171.x.

[12] B. Deng, S. Bouaziz, M. Deuss, A. Kaspar, Y. Schwartzburg,892 M. Pauly, Interactive design exploration for constrained893 meshes, Computer-Aided Design $61 \quad$ (2015) 13-23.894 doi:https://doi.org/10.1016/j.cad.2014.01.004.

[13] C. Jiang, C. Tang, M. Tomicic, H. Pottmann, J. Wallner, In-897 teractive Modeling of Architectural Freeform Structures Com-898 bining Geometry with Fabrication and Statics, in: P. Block,899 W. Wang, J. Knippers (Eds.), Advances in Architectural Ge-900 ometry, Springer, 2014, pp. 95-108. doi:https://doi.org/10.901 1007/978-3-319-11418-7\_7.

[14] R. Mesnil, C. Douthe, O. Baverel, Marionette Mesh: modelling903 free-form architecture with planar facets, International Journal904 of Space Structures 32 (3-4) (2017) 184-198. doi:https://doi.905 org/10.1177/0266351117738379.

[15] M. Bagneris, R. Motro, B. Maurin, N. Pauli, Structural Mor-907 phology issues in Conceptual Design of Double Curved Systems,908 International Journal of Space Structures 23 (2) (2008) 79-87.909 doi:https://doi.org/10.1260/026635108785260560. 910

[16] H. Pottmann, A. Asperl, M. Hofer, A. Kilian, Architecturalo11 Geometry, Bentley Institute Press, 2007.

[17] R. Mesnil, C. Douthe, O. Baverel, B. Léger, J.-F. Caron, Isog-913 onal moulding surfaces: A family of shapes for high node con-914 gruence in free-form structures, Automation in Construction 59 (2015) 38-47. doi:https://doi.org/10.1016/j.autcon.2015. 07.009.

[18] S. Krivoshapko, V. Ivanov, Encyclopedia of analytical surfaces, Springer, 2015. doi:10.1007/978-3-319-11773-7.

[19] R. Mesnil, Structural exploration of fabrication-aware design spaces for non-standard architecture, Ph.D. thesis, Université Paris-Est (2017) [cited February 14th 2018].

[20] H. Schober, Transparent shells - form topology structure, Ernst \& Sohn, 2016.

[21] C. Rogers, W. K. Schief, On the equilibrium of shell membranes under normal loading. Hidden integrability, Proceedings of the Royal Society of London A: Mathematical, Physical and Engineering Sciences 459 (2003) 2449-2462. doi:https://doi.org/10.1098/rspa.2003.1135.

[22] R. Mesnil, C. Douthe, O. Baverel, B. Léger, Generalised cyclidic nets for shape modelling in architecture, International Journal of Architectural Computing 15 (2) (2017) 148-168. doi:https: //doi.org/10.1177/1478077117714917.

[23] R. Martin, J. De Pont, T. Sharrock, Cyclide surfaces in computer aided design, The mathematics of surfaces (1986) 253268doi:https://doi.org/10.1016/0167-8396(90)90033-N.

[24] D. Dutta, R. Martin, M. Pratt, Cyclides in surface and solid modeling, IEEE Computer Graphics and Applications 13 (1) (1993) 53-59. doi:https://doi.org/10.1109/38.180118.

[25] A. Bobenko, E. Huhnen-Venedey, Curvature line parametrized surfaces and orthogonal coordinate systems: discretization with Dupin cyclides, Geometriae Dedicata 159 (1) (2012) 207-237. doi:https://doi.org/10.1007/s10711-011-9653-5.

[26] P. Bo, Y. Liu, C. Tu, C. Zhang, W. Wang, Surface fitting with cyclide splines, Computer Aided Geometric Designdoi:10.1016/j.cagd.2016.02.018.

[27] M. Bizzarri, M. Lávička, J. Vršek, Canal surfaces with rational contour curves and blends bypassing the obstacles, ComputerAided Design 64 (2015) 55-67. doi:10.1016/j.cad.2015.03. 002.

[28] K. Bana, K. Kruppa, R. Kunkli, M. Hoffmann, KSpheres an efficient algorithm for joining skinning surfaces, Computer Aided Geometric Design 31 (7-8) (2014) 499-509. doi:10.1016/j.cagd.2014.08.003.

[29] Y. Srinivas, D. Dutta, Intuitive procedure for constructing geometrically complex objects using cyclides, Computer-Aided Design 26 (4) (1994) 327-335. 
doi:10.1016/0010-4485(94)90078-7.

[30] M. J. Pratt, Quartic supercyclides I: Basic theory, Computer Aided Geometric Design 14 (7) (1997) 671-692. doi:https://doi.org/10.1016/S0167-8396(96)00057-X.

[31] A. Bobenko, P. Schröder, Discrete Willmore Flow, in: Proceedings of the third Eurographics symposium on Geometry processing, Eurographics Association, 2005, p. 101.

[32] C. Douthe, R. Mesnil, H. Orts, O. Baverel, Isoradial meshes: covering elastic gridshells with planar facets, Automation in Construction 83 (2017) 222-236. doi:https://doi.org/10. $1016 / j$. autcon. 2017.08.015.

[33] Z. Soyuçok, Infinitesimal deformations of surfaces and the stress distribution on some membranes under constant inner pressure, International Journal of Engineering Science 34 (9) (1996) 9931004. doi:https://doi.org/10.1016/0020-7225(95)00142-5.

[34] S. Timoshenko, J. Gere, Theory of elastic stability, McGrawHill, New York, 1961.

[35] D. P. Billington, The Tower and the Bridge: the new art of structural engineering, Princeton University Press, 1985.

[36] G. Elber, M.-S. Kim, The bisector surface of rational space curves, ACM Transactions on Graphics (TOG) 17 (1) (1998) 32-49. doi:https://doi.org/10.1145/269799.269801.

[37] S. Sechelmann, T. Rörig, A. Bobenko, Quasiisothermic mesh layout, in: Advances in Architectural Geometry, Springer, 2013, pp. 243-258. doi:https://doi.org/10.1007/ 978-3-7091-1251-9\_20.

[38] A. Vaxman, C. Müller, O. Weber, Conformal mesh deformations with Möbius transformations, ACM Transactions on Graphics (TOG) 34 (4) (2015) 55. doi:https://doi.org/10.1145/ 2766915 . 